\title{
Angular Effects in Proton-Induced Single-Event Upsets in Silicon-on-Sapphire and Silicon-on-Insulator Devices
}

\author{
S. D. Kniffin \\ Orbital Sciences Corporation \\ Dulles, VA 20166 USA
}

\author{
A. B. Sanders, R. A. Reed, and K. A. LaBel \\ NASA GSFC, Code 561.4 \\ Greenbelt, MD 20771 USA
}

M. S. Liu

Honeywell, SSEC

Plymouth, MN 55441 USA

\section{J. Tabbert}

Peregrine Semiconductor

Albuquerque, NM 87109 USA

\section{J. W. Swonger}

Peregrine Semiconductor

Melbourne, FL 32901 USA

\begin{abstract}
We present new data in the ongoing effort to bound the effect of proton angle of incidence on the single-event upset (SEU) rate in silicon-on-sapphire (SOS) and silicon-on-insulator (SOI) devices.
\end{abstract}

\section{INTRODUCTION}

Reed, et al. first predicted [1], [2] and later measured [3] the effect that the angle of particle incidence of an impinging proton has in the measured single-event upset (SEU) crosssection in silicon-on-sapphire (SOS) and silicon-on-insulator (SOI) devices. This effect manifests itself as an increase in measured SEU cross-section at "extreme" incidence angles. We present the data collected from three additional device types manufactured using these technologies. Two of the device types presented here also give us a first look at the effect of radiation hardening by design (RHBD) on SEU sensitivity in SOS and SOI devices. The primary concern, and therefore the driver behind this continuing research, is that if this effective increase in the total cross-section due to the change in the angle of incidence is not taken into account, the rate of errors predicted for a given device using standard methods could be low by as much as an order of magnitude.

\author{
J. F. McCabe, G. A. Gardner, J. Lintz, C. Ross, \\ K. Golke, and B. Burns \\ Honeywell, Defense and Space Electronics \\ Clearwater, FL 33764 USA
}

P. W. Marshall

Consultant

Brookneal, VA 24528 USA

\author{
H. S. Kim and J. D. Forney \\ Jackson and Tull \\ Washington, DC 20018 USA
}

\author{
M. A. Carts \\ Raytheon ITSS \\ Upper Marlboro, MD 20771 USA
}

\section{DeVICES TESTED}

The Peregrine PE926C31 and PE926C32 RS-422 line driver/receiver pair was manufactured using their Ultra Thin Silicon (UTSiTM) $0.5 \mu \mathrm{m}$ CMOS-on-sapphire process. Both the Honeywell HX6228 128k x 8 SRAM and the radiationhardened reprogrammable field programmable gate array (RHrFPGA) were manufactured on the RICMOSTM IV SOI $0.7 \mu \mathrm{m}$ process.

TABLE I. DEVICES TESTED

\begin{tabular}{|c|l|l|l|}
\hline $\begin{array}{c}\text { Part } \\
\text { Number }\end{array}$ & Manufacturer & Function & \multicolumn{1}{|c|}{$\begin{array}{l}\text { Design } \\
\text { Library }\end{array}$} \\
\hline $\begin{array}{l}\text { PE926C31 } \\
\text { PE926C32 }\end{array}$ & Peregrine & $\begin{array}{l}\text { RS-422 } \\
\text { Driver/Receiver }\end{array}$ & RHBD \\
\hline HX6228 & Honeywell & SRAM & $\begin{array}{l}\text { Standard } \\
\text { Honeywell } \\
\text { RHBD }\end{array}$ \\
\hline RHrFPGA & Honeywell & FPGA & $\begin{array}{l}\text { Improved } \\
\text { Honeywell } \\
\text { RHBD }\end{array}$ \\
\hline
\end{tabular}

This work was supported by the NASA Electronic Parts and Packaging (NEPP) Program, NASA Flight Projects, the Defense Threat Reduction Agency (DTRA) under IACRO 03-40351 and 04-40641, Honeywell SSEC, and Peregrine Semiconductor.
Presented by S. D. Kniffin at NSREC04 Data Workshop, Atlanta, GA, July 22, 2004

Page 1 of 4 


\section{TEST FACILITIES}

All devices were tested for proton-induced SEU at the Indiana University Cyclotron Facility (IUCF) with an incident $205 \mathrm{MeV}$ proton beam In addition, the Peregrine driverireceiver and the Honeywell SRAM were tested for proton-induced SEU at the University of California, Davis (UCD) Crocker Nuclear Laboratory (CNL) with a $63 \mathrm{MeV}$ proton beam.

\section{ANGLE CONVENTIONS}

Fig. 1 shows the representation of the angles as they are used here. Note that beam angle and angle of incidence are used interchangeably.
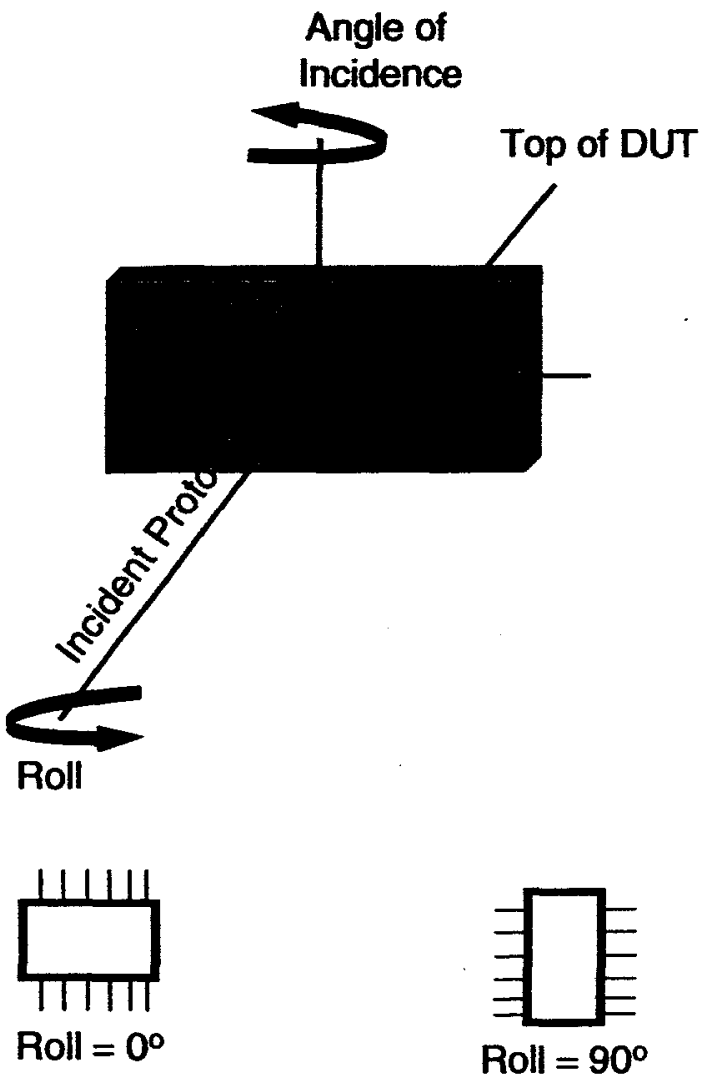

Angle of Incidence $=0^{\circ}$

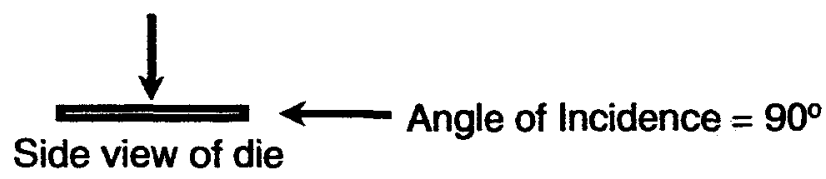

Fig. 1. Diagrams of angle conventions used.

\section{Device Description, Test SetuP AND Procedures}

\section{A. Peregrine PE926C31/PE926C32}

The PE926C31 and PE926C32 RS-422 driver/receiver pair is RHBD. Ohmic body contacts at dense spacings suppress any secondary charge collection that might occur. The receiver has two redundant comparators and a digital voter, unconnected in the data path, for the contingency that unacceptable SET performance was seen from the analog circuitry. Parasitic device capacitance is minimized, and the devices also oppose drive strength in the digital section.

For the test of the Peregrine RS-422 driver/receiver, a 20 $\mathrm{MHz}$ square wave input signal (clock) was run through a divide-by-2 prior to the device under test (DUT) for timing purposes. Delay Net 1 allows us to line up the DUT output with the reference signal in the comparator. Delay Net 2 allows us to line up the clock in the middle of each state in the square wave (the signal in the DUT is sampled on the rising edge of the clock signal, which is in the middle of the cycle in the DUT because of the divide-by-2). The signal is sampled at the midpoint of each high or low signal to determine if it had changed state; this prevents false eurors due to rising/falling edge timing errors. The error counter tallies non-compares. The functionality of the setup is verified by deliberately altering one of the Delay Nets or removing power from the DUT to induce errors.

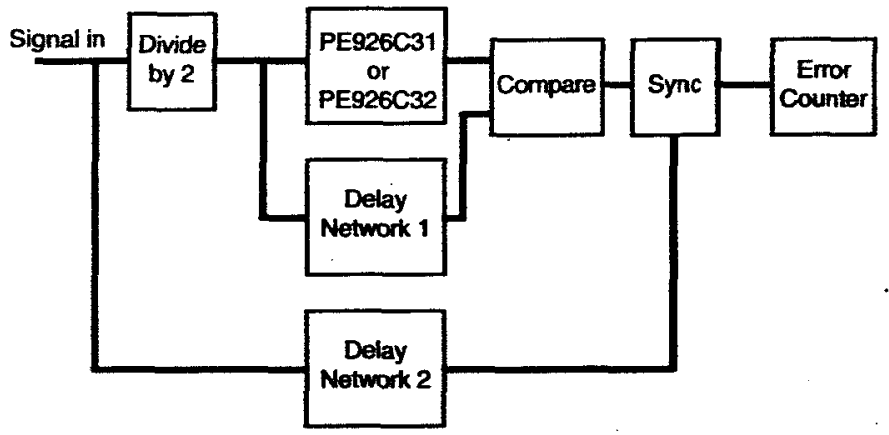

Fig. 2. Diagram of Peregrine PE926C31/PE926C32 test setup.

\section{B. Honeywell HX6228 SRAM}

The HX6228 is a rad-hard $\left(1 \mathrm{Mrad}\left(\mathrm{SiO}_{2}\right)\right)$ 1Mb SRAM designed for military applications and the space radiation environment. Typical read/write cycle times are $16 \mathrm{~ns}$ or less, and $25 \mathrm{~ns}$ or less across the full military temperature range. The RICMOSTM IV process is a $5 \mathrm{~V}$, SIMOX CMOS technology with a $150 \AA$ gate oxide and a minimum feature size of $0.7 \mu \mathrm{m}(0.55 \mu \mathrm{m}$ effective gate length). A seventransistor memory cell is used for single-event upset hardening, while three-layer metal power bussing and the low collection volume SIMOX substrate provide dose rate hardening.

In the Honeywell SRAM, a pattern of alternating 0's and 1's was written to the device prior to irradiation. The address counter initially cycles through each memory location while the predetermined data pattern is written. During irradiation, the data are read and rewritten continuously and then compared to a reference buffer. Once the comparison is completed, SEUs (incorrect data values) are written to FIFO 1 and the address location is captured and written to FIFO 2 when a misscompare is detected. Data and address information are then downloaded to a computer for analysis. A block diagram of the test setup is given below.

Presented by S. D. Kniffin at NSREC04 Data Workshop, Atlanta, GA, July 22, 2004 Page 2 of 4 


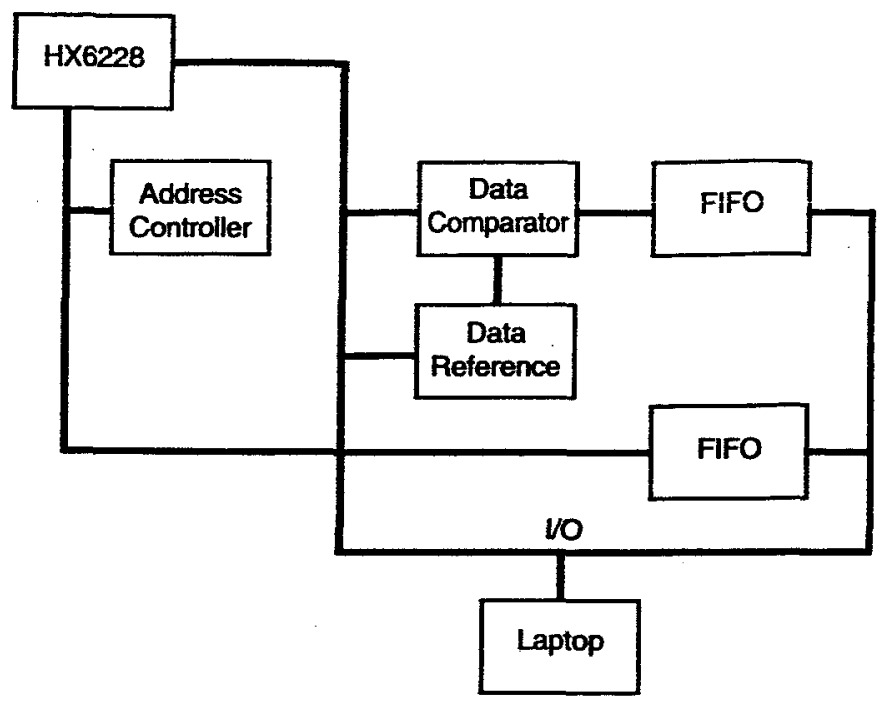

Fig. 3. Diagram of Honeywell HX6228 SRAM test setup.

\section{Honeywell RHrFPGA}

The RHrFPGA is an SRAM-based (reconfigurable) fieldprogrammable gate array manufactured by Honeywell using an RH SOI fabrication process. The RICMOSTM IV process is a $5 \mathrm{~V}$, SIMOX CMOS technology with a $150 \AA$ gate oxide and a minimum feature size of $0.7 \mu \mathrm{m}(0.55 \mu \mathrm{m}$ effective gate length). It has 6,400 user-configurable logic cells and 131,152 configuration SRAM cells. NASA GSFC funded the development, fabrication, and radiation testing of the RHrFPGA.

Proton testing was performed at IUCF on the RHrFPGA because it contains SRAM memory elements that were hardened after an earlier Honeywell SRAM that exhibited susceptibility to upset depending on proton angle of incidence. This sensitivity was attributed to a single secondary heavy ion hitting two transistors within a memory cell. The cross-section would be highest if the incident protons were parallel to the path between two sensitive transistors in a cell.

At IUCF, all irradiations were carried out at a $70^{\circ}$ angle of incidence at roll $=0^{\circ}$ and roll $=90^{\circ}$ because the configuration RAM and the application flip-flops are orthogonal to each other. The test was limited to $70^{\circ}$ because of constraints in rotating the fixture and concerns about irradiating the control device.

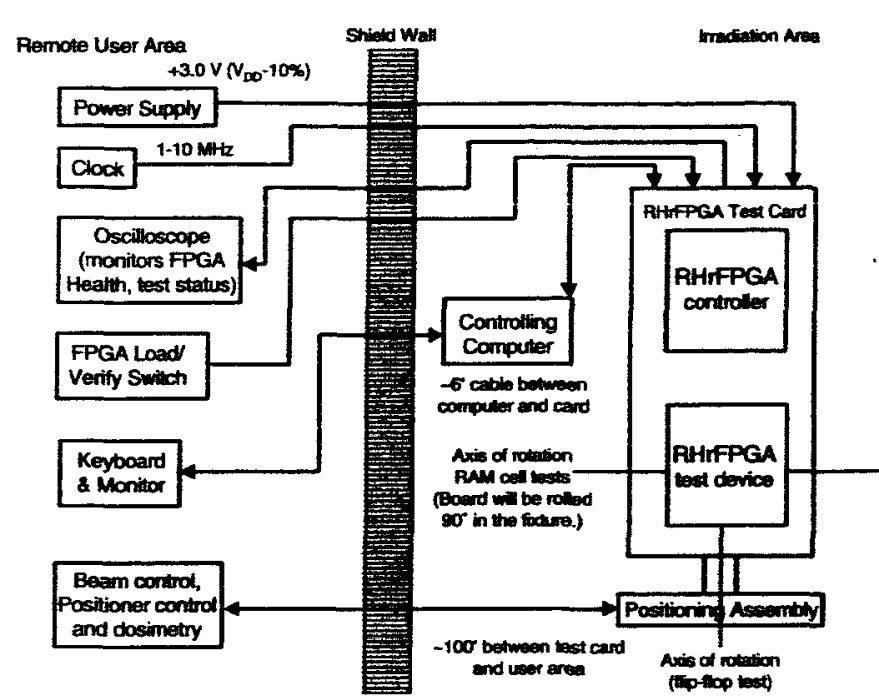

Fig. 4. Diagram of Honeywell RHrFPGA proton test setup at IUCF.

\section{TeSt Results}

\section{A. Peregrine PE926C31/PE926C32}

At IUCF, the devices were exposed to a $205 \mathrm{MeV}$ proton beam at a $90^{\circ}$ angle of incidence (grazing angle). The driver was only exposed at a roll of $0^{\circ}$ and the receiver was exposed at roll $=0^{\circ}$ and roll $=90^{\circ}$. No single-event transients (SETs) were observed to a fluence of $3.4 \times 10^{12} \mathrm{p} / \mathrm{cm}^{2}$ for each device type at IUCF.

At UCD, the devices were exposed to a $63 \mathrm{MeV}$ proton beam at a $90^{\circ}$ angle of incidence (grazing angle). Both device types were exposed at roll $=0^{\circ}$ and roll $=90^{\circ}$. No SETs were observed to a fluence of $1 \times 10^{13} \mathrm{p} / \mathrm{cm}^{2}$ for each device type at UCD.

\section{B. Honeywell HX6228 SRAM}

At IUCF, the devices were exposed to a $205 \mathrm{MeV}$ proton beam at roll $=0^{\circ}$ and roll $=90^{\circ}$ at a mumber of angles of incidence between $0^{\circ}$ and just over $90^{\circ}$. The results showed an angle of incidence effect on the order of a factor of 2 increase in proton cross-section with angle. There was little difference in the results for either roll direction (see Fig. 5-7). The cross sections given are in $\mathrm{cm}^{2} /$ device.

At UCD, the devices were exposed to a $63 \mathrm{MeV}$ proton beam at roll $=0^{\circ}$ and roll $=90^{\circ}$ at a number of angle of incidence between $0^{\circ}$ and just over $90^{\circ}$. The results at UCD present a very different picture. In the case of the condition of $0^{\circ}$ roll, there was only one SEU captured with the beam perpendicular to the die and again only one SEU with the beam grazing the surface of the die in this orientation In the $90^{\circ}$ roll condition, there is more than an order of magnitude difference in the cross section compared to the $0^{\circ}$ roll (see Fig. 8). These data would imply an interesting geometry in the sensitive volume that should be studied in more detail, including circuit simulation. 


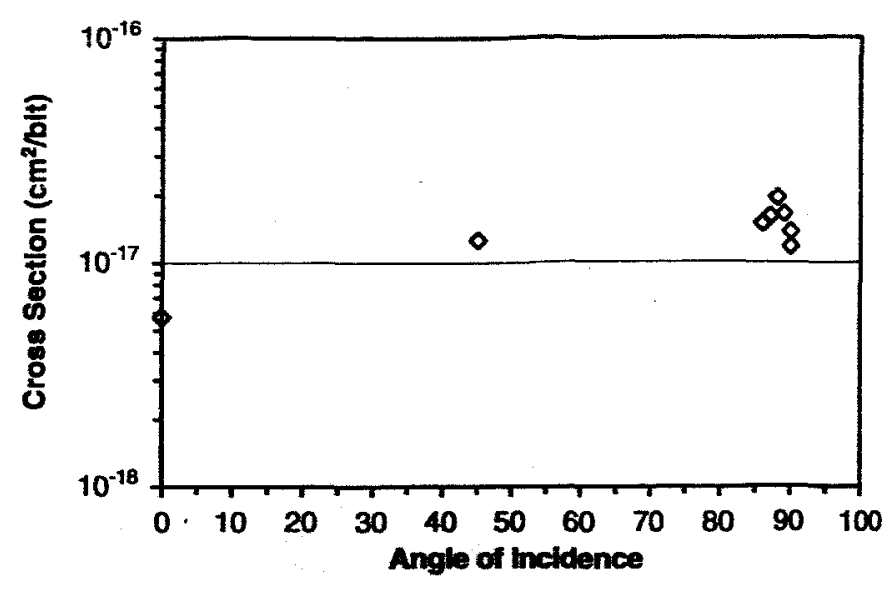

Fig. 5. IUCF results for DUT 2 at $0^{\circ}$ roll (205MeV protons).

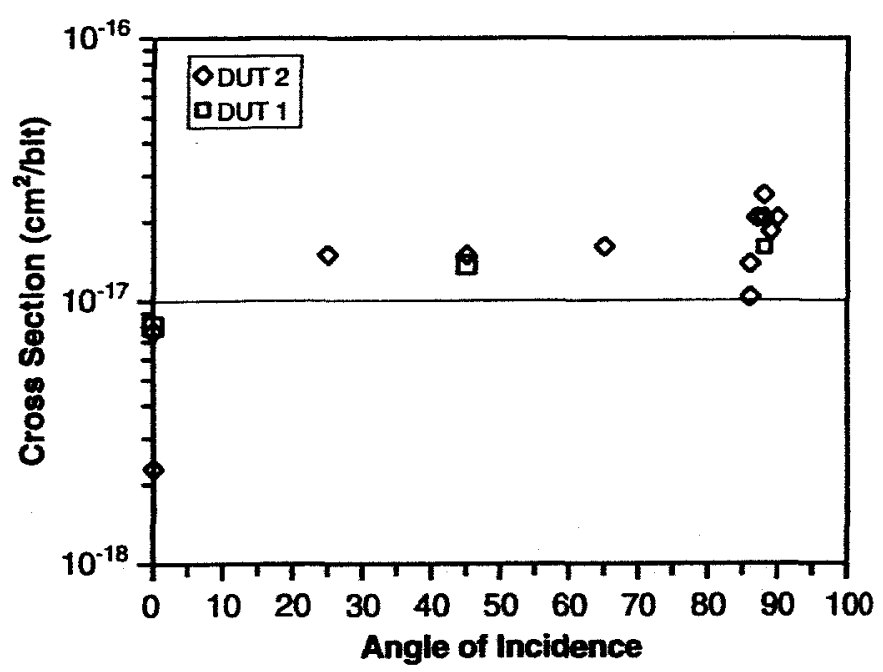

Fig. 6. IUCF results for DUTs 1 and 2 at $90^{\circ}$ roll (205MeV protons).

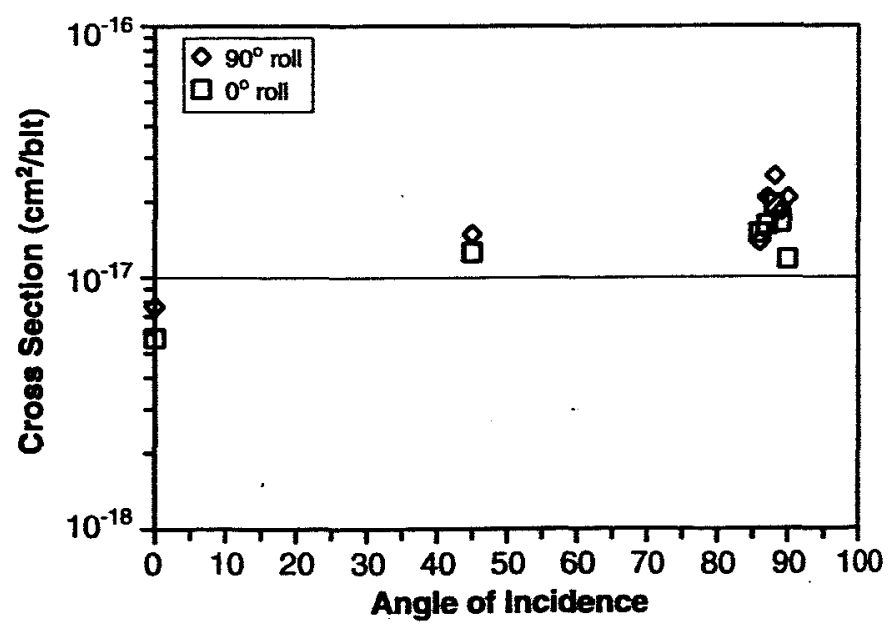

Fig. 7. IUCF results comparing $0^{\circ}$ and $90^{\circ}$ roll data (205MeV protons).

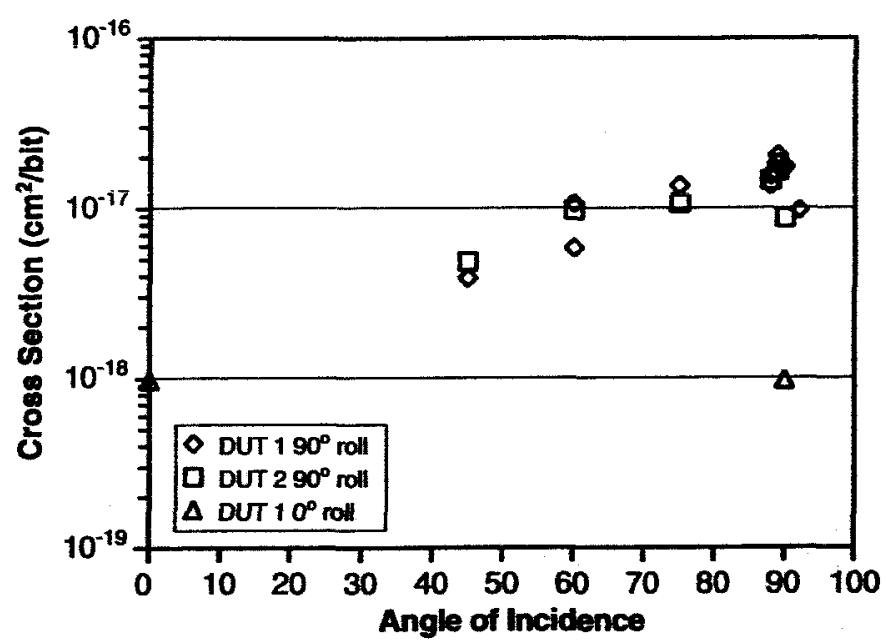

Fig. 8. UCD results comparing $0^{\circ}$ and $90^{\circ}$ roll data ( $63 \mathrm{MeV}$ protons).

\section{Honeywell RHrFPGA}

At IUCF, the devices were exposed to a $205 \mathrm{MeV}$ proton beam. All exposures were performed at a $70^{\circ}$ angle of incidence in the roll $=0^{\circ}$ and roll $=90^{\circ}$ test configurations. No SEUs were observed to a fluence of $3.4 \times 10^{13} \mathrm{p} / \mathrm{cm}^{2}$ in both test configurations.

\section{Discussion}

Our results show that RHBD best practices can significantly decrease SEU sensitivity of SOS and SOI devices, in some cases eliminating proton-induced SEU sensitivity. The results also indicate that for some devices, the roll of the DUT can impact proton sensitivity and may have implications for heavy-ion irradiation as well. In some cases, this roll effect can be greater in magnitude than the previously observed enhancements of proton SEU cross-section seen for protons at grazing angle of incidence. These results have significant implications for both testing and rate prediction.

It should be noted that the Peregrine devices tested were only operated at $20 \mathrm{MHz}$. The potential for increased SEU sensitivity at much higher speeds needs to be examined.

\section{ACKNOWLEDGMENT}

The authors would like to acknowledge the sponsors of this effort: a portion of the NASA Electronic Parts and Packaging (NEPP) Program, NASA Flight Projects, the Defense Threat Reduction Agency (DTRA) under IACRO 03-40351 and 0440641, Honeywell SSEC, and Peregrine Semiconductor.

\section{REFERENCES}

[1] R. A. Reed, P. J. McNulty, and W. G. Abdel-Kader, "Implications of angle of incidence in SEU testing of modern circuits," IEEE Trans. Nucl. Sci., vol. 41, pp. 2049-2054, Dec. 1994.

[2] R. A. Reed and P. J. McNulty, "Effects of geometry on the proton SEU dependence on the angle of incidence," IEEE Trans. Nucl. Sci., vol. 42, pp. 1803-1808, Dec. 1995.

[3] R. A. Reed, et al., "Evidence for angular effects in proton-induced single-event upsets," IEEE Trans. Nuc. Sci., vol. 49, no. 6, pp. 30383044, Dec. 2002.

Presented by S. D. Kniffin at NSREC04 Data Workshop, Atlanta, GA, July 22, 2004

Page 4 of 4 